\title{
Intranasal delivery of bone marrow stromal cells to spinal cord lesions
}

\author{
Koshi Ninomiya, MD, Koichi Iwatsuki, MD, PhD, Yu-ichiro Ohnishi, MD, PhD, \\ Toshika Ohkawa, MD, PhD, and Toshiki Yoshimine, MD, PhD \\ Department of Neurosurgery, Osaka University Graduate School of Medicine, Suita City, Osaka, Japan
}

\begin{abstract}
OBJECT The intranasal delivery of bone marrow stromal cells (BMSCs) or mesenchymal stem cells to the injured brains of rodents has been previously reported. In this study, the authors investigated whether BMSCs migrate to spinal cord lesions through an intranasal route and whether the administration affected functional recovery.

METHODS Forty Sprague-Dawley rats that were subjected to spinal cord injuries at the T7-8 level were divided into 5 groups (injured + intranasal BMSC-treated group, injured + intrathecal BMSC-treated group, injured-only group, injured + intranasal vehicle-treated group, and injured + intrathecal vehicle-treated group). The Basso-Beattie-Bresnahan (BBB) scale was used to assess hind limb motor functional recovery for 2 or 4 weeks. Intralesionally migrated BMSCs were examined histologically and counted at 2 and 4 weeks. To evaluate the neuroprotective and trophic effects of BMSCs, the relative volume of the lesion cavity was measured at 4 weeks. In addition, nerve growth factor (NGF) and brain-derived neurotrophic factor (BDNF) levels in the CSF were evaluated at 2 weeks.
\end{abstract}

RESULTS Intranasally administered BMSCs were confirmed within spinal cord sections at both 2 and 4 weeks. The highest number, which was detected in the intrathecal BMSC-treated group at 2 weeks, was significantly higher than that in all the other groups. The BBB score of the intranasal BMSC-treated group showed statistically significant improvements by 1 week compared with the control group. However, in the final BBB scores, there was a statistically significant difference only between the intrathecal BMSC-treated group and the control group. The cavity ratios in the BMSC-treated groups were smaller than those of the control groups, but the authors did not find any significant differences in the NGF and BDNF levels in the CSF among the treatment and control groups.

CONCLUSIONS BMSCs reached the injured spinal cord through the intranasal route and contributed to the recovery of hind limb motor function and lesion cavity reduction. However, the effects were not as significant as those seen in the intrathecal BMSC-treated group.

http://thejns.org/doi/abs/10.3171/2014.10.SPINE14690

KEY WORDS intranasal delivery; bone marrow stromal cells; spinal cord injury

$\mathrm{B}$ ONE marrow stromal cells (BMSCs), which are also called mesenchymal stem cells (MSCs), are a promising resource for the treatment of spinal cord injury (SCI). Their contribution to neurological functional recovery has been previously reported. ${ }^{19-21,26}$

BMSCs, which are harvested from bone marrow, proliferate easily and quickly. They have the potential to differentiate into neurons, ${ }^{16,27}$ but their secretion of trophic factors and cytokines is thought to be very important for neuroprotection, axonal growth, and tissue repair within the injured spinal cord.,3,19-21,26 Moreover, the homing ability of BMSCs toward the injured site has been noted. ${ }^{14}$ Therefore, the effectiveness of the intralesional, ${ }^{3}$ intraarterial, ${ }^{21}$ intravenous, ${ }^{18,19}$ and intrathecal ${ }^{2}$ routes for the administration of BMSCs to the lesion site has been previously tested.

In clinical use, it is very important when selecting treatment routes to consider invasiveness and simplicity as

ABBREVIATIONS BBB = Basso-Beattie-Bresnahan; BDNF = brain-derived neurotrophic factor; BMSC = bone marrow stromal cell; DAPI = 4',6-diamidino-2-phenylindole; DMEM = Dulbecco modified Eagle medium; EGFP = enhanced green fluorescent protein; i.n. = intranasal; i.t. = intrathecal; MSC = mesenchymal stem cell; NGF = nerve growth factor; PBS = phosphate-buffered saline; $\mathrm{SCl}=$ spinal cord injury; $\mathrm{SEM}=$ standard error of the mean .

SUBMITTED July 11, 2014. ACCEPTED October 24, 2014.

INCLUDE WHEN CITING Published online April 3, 2015; DOI: 10.3171/2014.10.SPINE14690.

DISCLOSURE This study was supported in part by a grant-in-aid for exploratory research (No. 10) from the Osaka University Medical Center for Translational and Clinical Research. 
well as effectiveness. Recently, the intranasal administration of BMSCs into lesioned brains of rodents has been reported. $5,9,12,13,22,24$ After their administration by simple drops into the nostrils, BMSCs are thought to migrate into the brains through the olfactory nerve route or trigeminal ganglion route. From the point of view of minimizing invasiveness, the intranasal route is thought to be the least invasive of all the routes mentioned above.

To the best of our knowledge, this is the first report of an evaluation of intranasal BMSC administration to the injured spinal cord.

\section{Methods \\ Animals}

All animals were handled in accordance with the guidelines of the Laboratory Animals Care and Use Committee of the Osaka University Faculty of Medicine.

All rats were purchased from Japan SLC, Inc. The rats were provided food and water ad libitum and kept in a 12hour light/dark cycle throughout the study.

\section{BMSC Isolation, Culture, and Characterization}

To prepare the BMSCs, two 8-week-old enhanced green fluorescent protein (EGFP) Sprague-Dawley male rats (Japan SLC, Inc.) were used.

After the rats were deeply anesthetized by an intraperitoneal overdose injection of medetomidine hydrochloride, midazolam, and butorphanol tartrate, the femurs and tibias were aseptically removed, and the bone marrow was flushed from each piece with $10 \mathrm{ml}$ of Dulbecco modified Eagle medium (DMEM, Life Technologies Corp.) with a sterile syringe. After filtrating with a $100-\mu l$ cell strainer (BD Falcon, BD Biosciences), the bone marrow was spun at $300 \times g$ for 5 minutes at room temperature.

The collected cells were placed in $75-\mathrm{cm}^{2}$ culture flasks and cultured with DMEM supplemented with $10 \%$ fetal calf serum, $100 \mu \mathrm{g} / \mathrm{ml}$ streptomycin, and $100 \mu \mathrm{g} / \mathrm{ml}$ penicillin. The next day, the culture medium was changed to remove nonadherent cells. The culture medium was subsequently changed twice a week. The cells were passaged when they reached confluence. The cells of passage 2 were used for all the experiments.

The cells were characterized by the expression of specific surface antigens by using immunohistochemistry with antibodies against CD90 (+), CD11b (-), CD31 (-), and CD45 (-) (Fig. 1). The following antibodies were applied: a mouse monoclonal antibody against CD90 (1:100, 554892, BD Pharmingen, BD Biosciences), a mouse monoclonal antibody against CD11b (1:100, ab1211, abcam plc), a mouse monoclonal antibody against CD31 (1:100, ab24590, abcam plc), and a rabbit polyclonal antibody against CD45 (1:100, ab10558, abcam plc). For secondary antibodies, an Alexa Fluor goat anti-mouse 546-conjugated IgG (1:200, Life Technologies Corp.) and an Alexa Fluor donkey anti-rabbit 546-conjugated IgG (1:500, Life Technologies Corp.) were used.

\section{Surgical Procedures}

Forty-five 8-week-old male Sprague-Dawley rats were deeply anesthetized by the inhalation of $2 \%$ to $3 \%$ isoflu- rane in air with a flow of $2 \mathrm{~L} /$ minute. A laminectomy was performed to expose the T7-8 spinal cord segments, and a crushing injury was made at that level with an SCI device (180 kdyn, 5 seconds; IH impactor; Muromachi Kikai Co., Ltd.). The wound was closed in 2 layers. Flomoxef sodium $(50 \mathrm{mg} / \mathrm{kg}$; Shionogi \& Co., Ltd.) was injected subcutaneously after the surgery to prevent urinary tract infections. Animals had their bladders manually emptied twice daily until the recovery of micturition, and they were carefully inspected for weight loss and dehydration.

Because 5 rats showed hind limb movements 6 hours after surgery, they were excluded from this experiment.

The day after surgery, all rats with a score of zero on the Basso-Beattie-Bresnahan (BBB) scale $(n=40)$ were divided into 5 groups $(n=8)$, and the treatments were performed.

For the BMSC treatment groups, an injured + intranasal (i.n.) BMSC-treated group and an injured + intrathecal (i.t.) BMSC-treated group were prepared. For the control groups, an injured-only group, an injured + i.n. vehicletreated group, and an injured + i.t. vehicle-treated group were prepared.

\section{Intranasal Administration of Cells/Vehicle}

This procedure was done by inserting a $1-\mathrm{ml}$ syringe and a 27-gauge blunt needle up to $5 \mathrm{~mm}$ into each nostril of each rat. After anesthetization by isoflurane inhalation, as previously mentioned, the rats were held by a hand grip to immobilize the skull.

Prior to the cell treatment, the rats were given $100 \mathrm{U}$ of hyaluronidase (SERVA Electrophoresis $\mathrm{GmbH}$ ) dissolved in $40 \mu \mathrm{l}$ of sterile phosphate-buffered saline (PBS) in both nostrils in accordance with the literature ${ }^{9}$ to promote permeability. Thirty minutes later, the BMSC suspension (2 $\times 10^{5}$ BMSCs in $40 \mu \mathrm{l}$ of sterile PBS) was applied twice to each nostril with an alternating application of $10-\mu l$ drops. The vehicle-treated group was given $40 \mu l$ of sterile PBS.

\section{Intrathecal Administration of Cells/Vehicle}

While under anesthetization with isoflurane inhalation as previously mentioned, the rats were positioned with their necks bent anteriorly. The cisterna magna was punctured with a 27-gauge needle. Proper placement of the needle was indicated by the presence of CSF in the needle hub. Then, the BMSC suspension $\left(2 \times 10^{5}\right.$ BMSCs in $40 \mu \mathrm{l}$ of sterile PBS) was slowly injected over 1 minute. The vehicle-treated group was administered $40 \mu \mathrm{l}$ of sterile PBS.

\section{Assessment of Hind Limb Locomotor Behavior}

The BBB locomotor rating scale was used to assess hind limb locomotor behavior. The rats were observed walking freely in an open field for 10 minutes with video monitoring twice a week. Half of the rats were observed for 2 weeks, and the rest were observed for 4 weeks. Blinded observers assessed the scores.

\section{Preparation of the SCI Sections}

Half of the rats in each group were randomly selected and sacrificed 14 days postsurgery, and the rest were sacrificed 28 days postsurgery. They were killed by means 


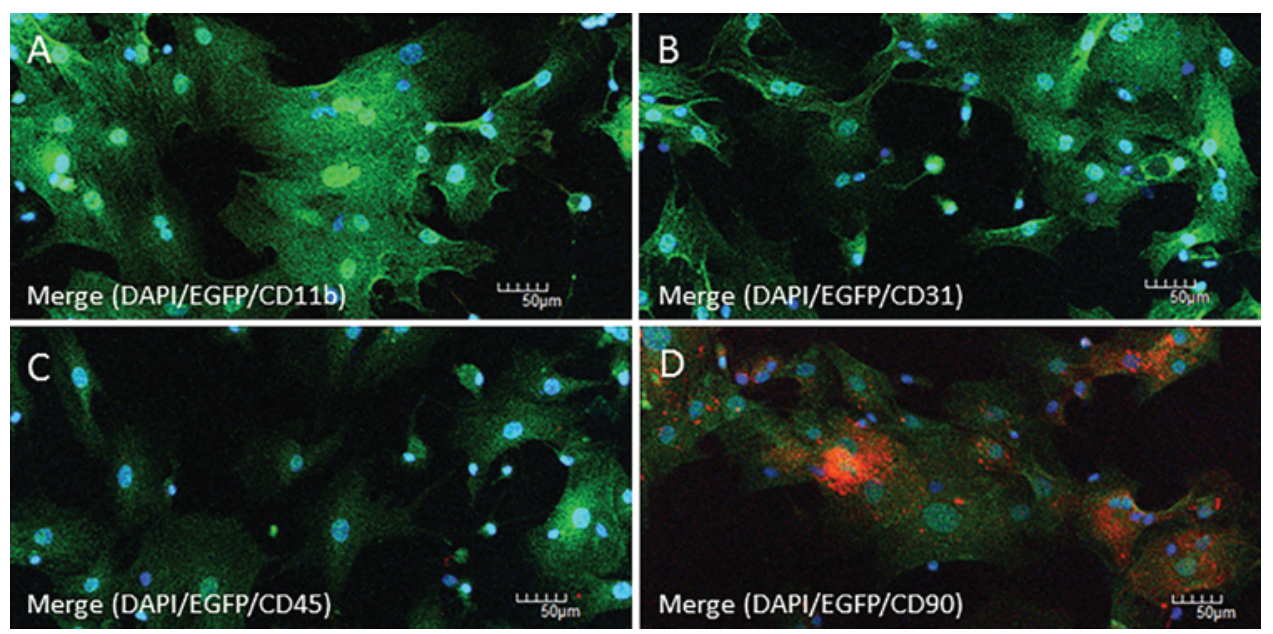

FIG. 1. Immunohistochemical characterization of passage-2 BMSCs in culture. Immunostaining with DAPI (blue), EGFP (green), CD11b (red), CD31 (red), CD45 (red), and the merged images are shown. CD11b (A), CD31 (B), and CD45 (C) immunostaining were negative, and CD90 (D) immunostaining was positive. Bar $=50 \mu \mathrm{m}$.

of an intraperitoneal overdose injection of medetomidine hydrochloride, midazolam, and butorphanol tartrate. They were then perfused with PBS, followed by $4 \%$ paraformaldehyde. The spinal cords were rapidly dissected and cryoprotected in $4 \%$ paraformaldehyde for 24 hours, which was followed by $30 \%$ sucrose for 24 hours. Then, tissue blocks that were $8 \mathrm{~mm}$ long $(4 \mathrm{~mm}$ rostrally and caudally from the epicenter) were taken. Transverse cryosections that were $10 \mu \mathrm{m}$ thick were prepared.

\section{Fluorescent Immunohistochemistry for the Quantification of the BMSCs}

Every third section from the epicenter in the rostral and caudal directions was selected, and 20 sections were used in each of the animals. They were coated with mounting medium containing 4',6-diamidino-2-phenylindole (DAPI; VECTASHIELD, Vector Laboratories, Inc.). The total number of BMSCs was measured by counting the EGFP and DAPI double-positive cells that were visualized with fluorescence microscopy, and the mean BMSC counts of each group were compared statistically. To confirm whether EGFP and DAPI double-positive cells were really BMSCs, immunostaining with CD90 (a mouse monoclonal antibody against CD90, 1:100, 554892, BD Pharmingen, BD Biosciences) of the other 5 spinal cord sections of every animal in the i.n. BMSC-treated group was added. An Alexa Fluor goat anti-mouse 546-conjugated IgG (1:200, Life Technologies Corp.) was used as a secondary antibody, and the sections were observed with fluorescence microscopy.

In addition, in the i.n. BMSC-treated group, $20 \mathrm{sec}-$ tions were cut $4 \mathrm{~mm}$ apart from the epicenter both rostrally and caudally. The sections were coated in the same way as described above and assessed for the presence of BMSCs.

\section{Hematoxylin and Eosin Staining for Lesion Cavity Analysis}

A total of 20 sections that included every third section that was collected from the epicenter in the rostral and caudal directions were used from 20 rats that were sacrificed 28 days postsurgery. We performed hematoxylin and eosin staining to analyze the extent of the cystic lesion. The mean cavity ratio of each rat was calculated by dividing the measured injured cavity area by the entire spinal cord slice manually with ImageJ software (version 1.47; http://rsbweb.nih.gov/ij/index.html). A mean ratio was calculated for each group, and between-groups differences in the ratios were analyzed for statistical significance.

The cavity ratios and final BBB scores of all 20 rats at 4 weeks were compared with a logistic regression analysis by using JMP Pro 10 (http://www.jmp.com/en_us/soft ware/jmp-pro.html).

\section{GAP-43 Fluorescent Immunohistochemistry for Analysis of Axonal Regrowth}

To analyze axonal regrowth at 4 weeks, 5 spinal cord sections from each rat were selected and immunostained with rabbit polyclonal antibody against GAP-43 (1:500, ab16053, abcam plc), which was followed by an Alexa Fluor donkey anti-rabbit 546-conjugated IgG (1:500, Life Technologies Corp.), which was used as a secondary antibody. The sections were then observed with fluorescence microscopy.

\section{ELISA of the NGF and BDNF Levels in CSF}

To evaluate the neurotrophic-productive ability of BMSCs, CSF samples (100 $\mu \mathrm{l} /$ animal) from 3 groups (injured + i.n. BMSC-treated group, injured + i.t. BMSCtreated group, and the injured-only group; $n=5$ ) were carefully collected 2 weeks after the surgery by cisterna magna puncture so that the CSF was not mixed with blood. Centrifugation was done at $9000 \times g$ for 15 minutes, and the supernatant was used. The NGF and BDNF concentrations were examined with ELISA kits (NGF Emax ImmunoAssay System and Promega/BDNF Emax ImmunoAssay System, Promega Corp.) according to the manufacturer's protocol. 


\section{Statistical Analyses}

The statistical analyses were performed with 1-way analysis of variance (ANOVA), which was followed by a Tukey multiple comparison test with JMP Pro 10; $p$ values less than 0.05 were considered statistically significant. The results are expressed as the mean \pm standard error of the mean (SEM).

\section{Results}

\section{BMSC Migration Into Spinal Cord Lesions}

The mean BMSC counts are shown in Fig. 2E. There were no DAPI and EGFP double-positive cells in the control groups. However, DAPI and EGFP double-positive cells were observed around the lesion cavities in the i.n. BMSC-treated group at both 2 weeks (Fig. 2C; $80.00 \pm$ $29.67)$ and 4 weeks $(94.75 \pm 26.75)$. But there were no DAPI and EGFP double-positive cells in sections obtained $4 \mathrm{~mm}$ from the SCI epicenter in either the rostral or caudal direction.

The highest number of BMSCs was detected in the i.t. BMSC-treated group 14 days postsurgery (328.25 \pm
48.01); this mean value was significantly greater than the mean values in any other group. In particular, the cells penetrated deeply around the lesion cavities in this group (Fig. 2B). In addition, statistically significant differences in the number of BMSCs that had migrated at 4 weeks were seen in this group in comparison with the control groups.

There were DAPI and EGFP double-positive cells with CD90 positivity, and these cells were confirmed as BMSCs in the i.n. BMSC-treated group (Fig. 2D).

\section{Hind Limb Locomotor Functional Recovery}

The BBB scores of the groups that were observed at 2 weeks $(n=8)$ are shown in Fig. 3A. A statistically significant increase was observed in the i.n. BMSC-treated group $(7.0 \pm 0.68)$ and the i.t. BMSC-treated group (7.5 \pm 1.32 ) at 7 days compared with the i.t. vehicle-treated group (1.75 \pm 0.73$)$. However, at 2 weeks, significant differences were observed only between the i.t. BMSC-treated group $(9.25 \pm 1.53)$ and the control groups (the injuredonly group and the i.t. vehicle-treated group) $(\mathrm{p}<0.05)$.

The BBB scores of the groups that were observed at
A

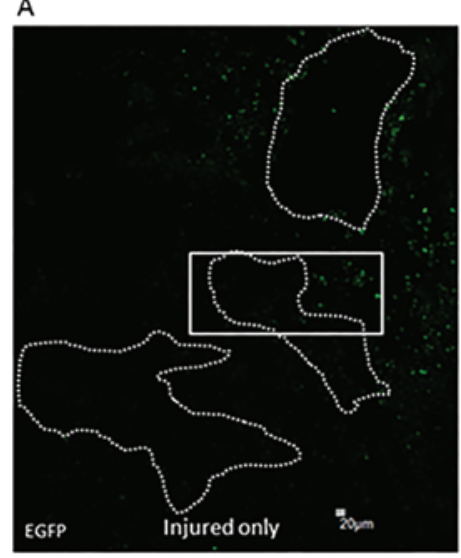

B

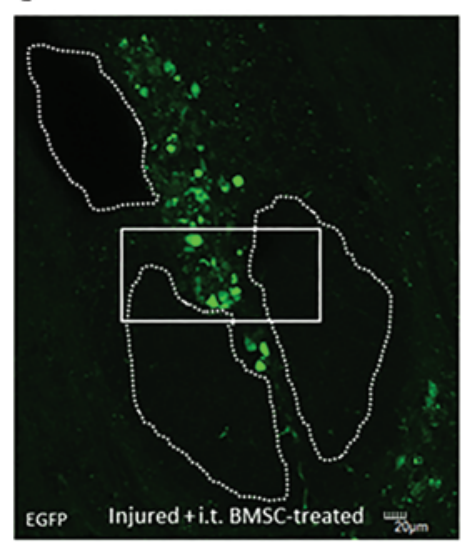

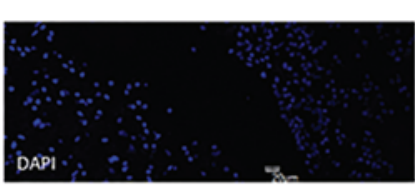
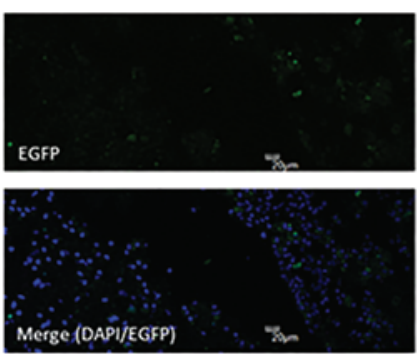

Merge (DAPIEGFP)
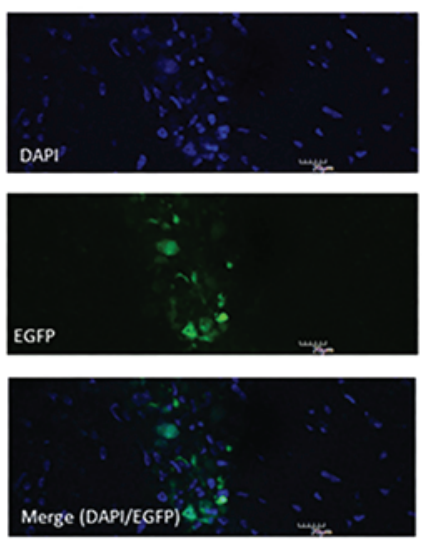

C
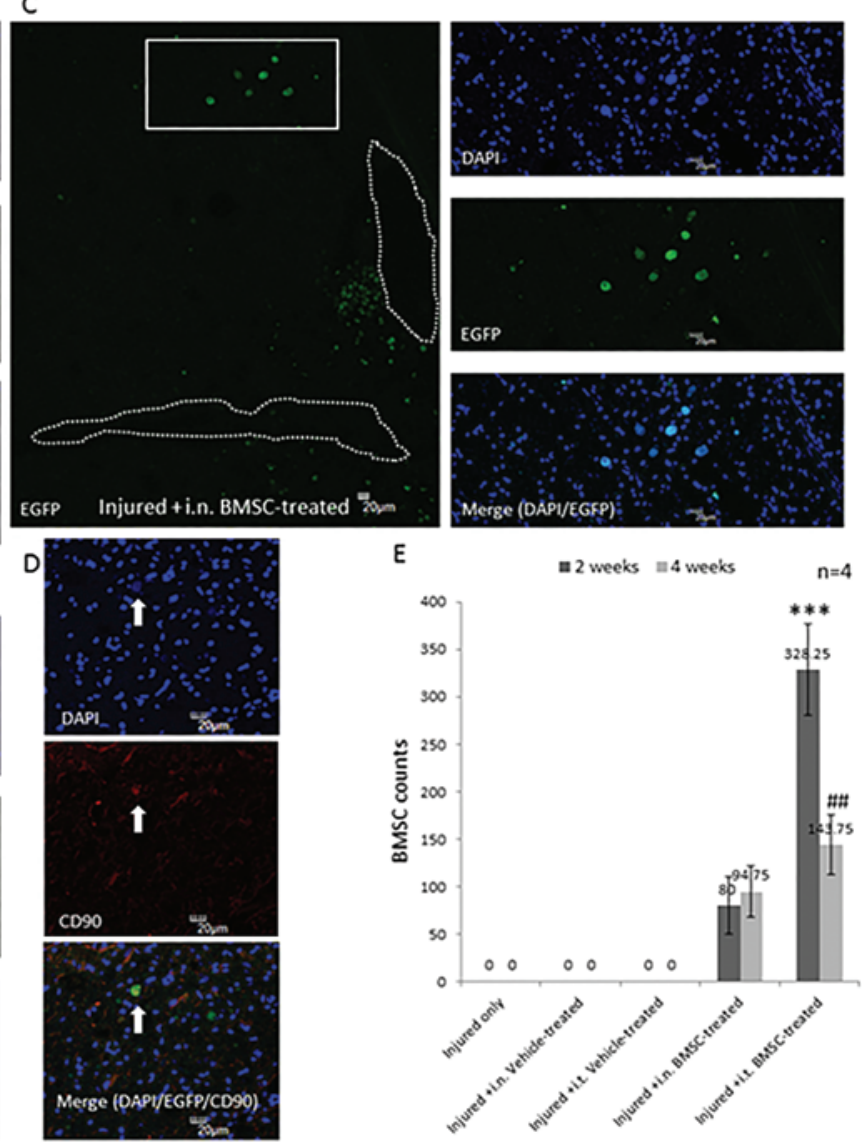

E

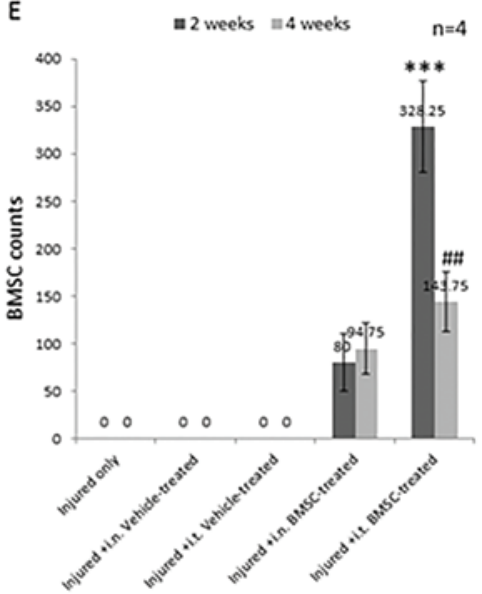

FIG. 2. A-D: Fluorescent images of transverse lesion sections obtained from animals killed 2 weeks after injury. The regions within the boxes in A, B, and C are enlarged at the right. DAPI (blue) and EGFP (green) double-positive cells were confirmed in both the i.n. and i.t. BMSC-treated groups (B and $C$ ), whereas there were none in the control group (A). DAPI and EGFP doublepositive cells (arrow) with CD90 (red) positivity in the i.n. BMSC-treated group are shown (D). E: Migrated BMSC counts (mean \pm SEM). The highest number was found in the i.t. BMSC-treated group at 2 weeks. ${ }^{* *} p<0.001$, significant difference for the i.t. BMSC-treated group at 2 weeks versus all the other groups. \#\#p $<0.01$, significant difference for the i.t. BMSC-treated group at 4 weeks versus all the control groups. Bar $=20 \mu \mathrm{m}$. The dots indicate the lesion cavity. 


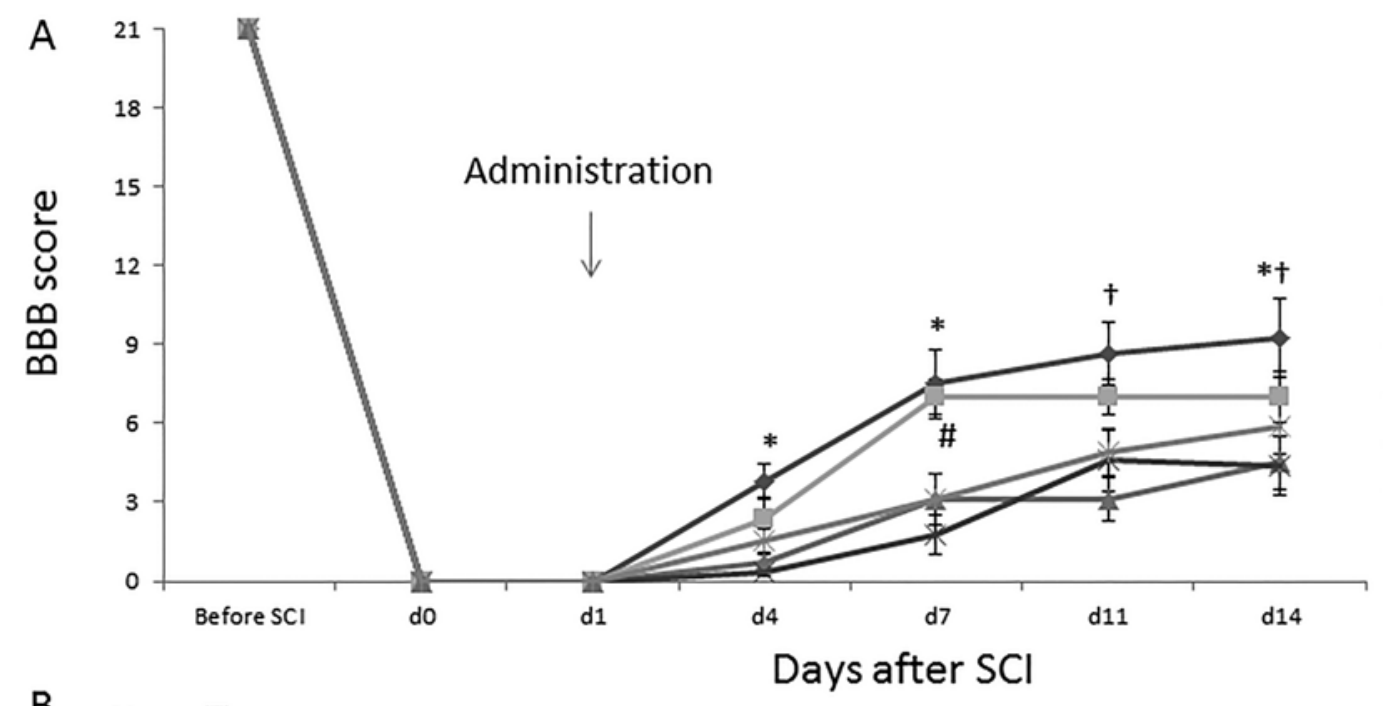

$$
n=8
$$

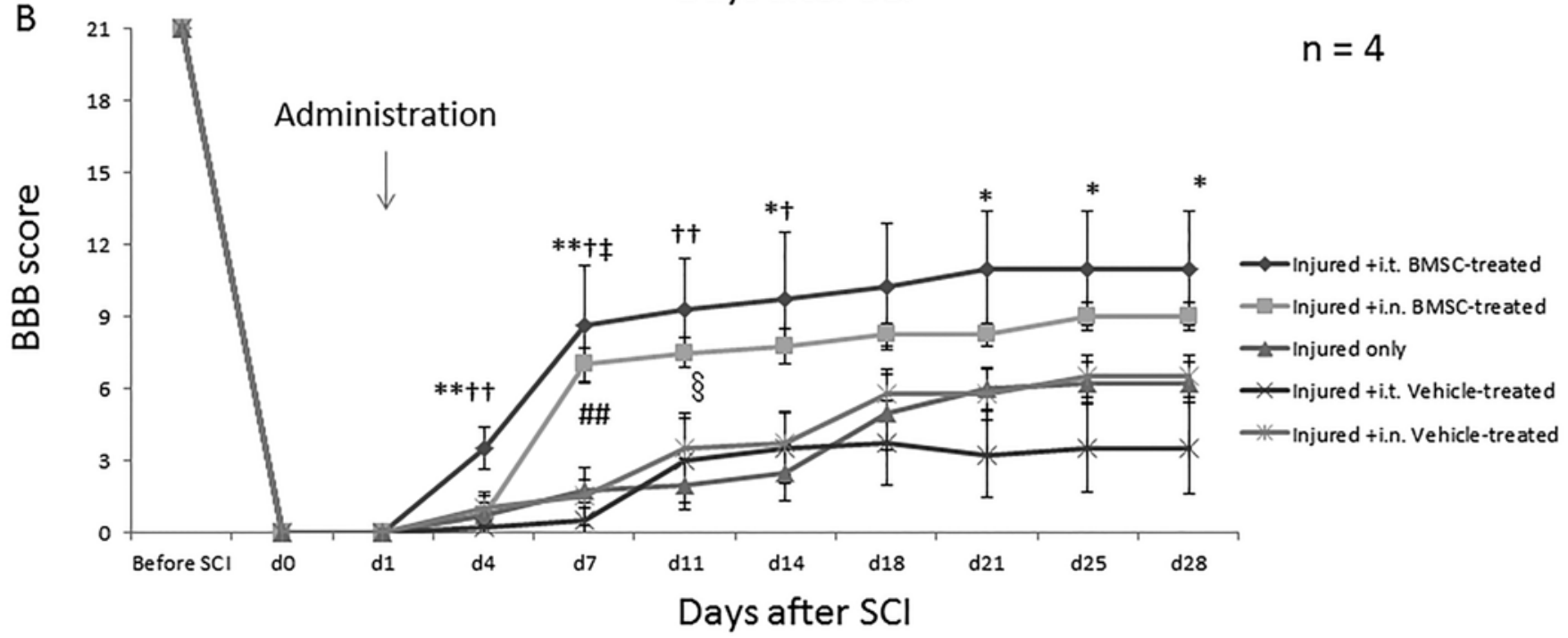

FIG. 3. A: BBB scores in the groups observed at 2 weeks $(n=8$; mean \pm SEM). The i.n. BMSC-treated group showed statistically significant improvements at Day 7. B: BBB scores in the groups observed at 4 weeks $(n=4$; mean \pm SEM). For the final BBB score, only the i.t. BMSC-treated group showed a statistically significant improvement. ${ }^{*} p<0.05,{ }^{* *} p<0.01$, significant difference for the i.t. BMSC-treated group versus the i.t. vehicle-treated group. $\uparrow p<0.05, \uparrow \uparrow p<0.01$, significant difference for the i.t. BMSC-treated group versus the injured-only group. $\# p<0.05, \# \# p<0.01$, significant difference for the i.n. BMSC-treated group versus the i.t. vehicle-treated group. $\neq p<0.05$, significant difference for the i.t. BMSC-treated group versus the i.n. vehicle-treated group. $\S p<0.05$, significant difference for the i.n. BMSC-treated group versus the injured-only group.

4 weeks $(n=4)$ are shown in Fig. 3B. There was no statistically significant increase in the BBB score of the i.n. BMSC-treated group after 2 weeks. The final BBB scores in the i.n. BMSC-treated, the i.t. BMSC-treated, and the i.t. vehicle-treated groups were $11 \pm 2.38,9 \pm 0.58$, and $3.5 \pm 1.89$, respectively. Finally, a significant difference was found only between the i.t. BMSC-treated group and the i.t. vehicle-treated group $(\mathrm{p}<0.05)$.

\section{Spinal Cord Lesion Cavity Ratio}

The results are shown in Fig. 4. The cavity ratios in the BMSC-treated groups were smaller than those in the control groups. However, a significant difference was observed only between the i.t. BMSC-treated group (21.86 \pm $2.14 \%)$ and the i.n. vehicle-treated group $(38.00 \pm 5.67 \%)$ $(\mathrm{p}<0.05)$ (Fig. 4D). A negative correlation was found between the final BBB scores at 4 weeks and the cavity ratio in the regression analysis $\left(\mathrm{R}^{2}=0.33, \mathrm{p}=0.0076\right)$ (Fig. 4E).

\section{Axonal Regrowth}

According to the anti-GAP-43 immunostaining, very little to no axonal regrowth was present in the control groups (data not shown). However, some axonal regrowth was observed near the migrated BMSCs in the BMSCtreated groups (Fig. 5A).

\section{Quantification of NGF and BDNF Levels in the CSF}

As shown in Fig. 5B, the NGF concentrations (ng/ml) in the CSF of the i.n. BMSC-treated group, the i.t. BMSC- 

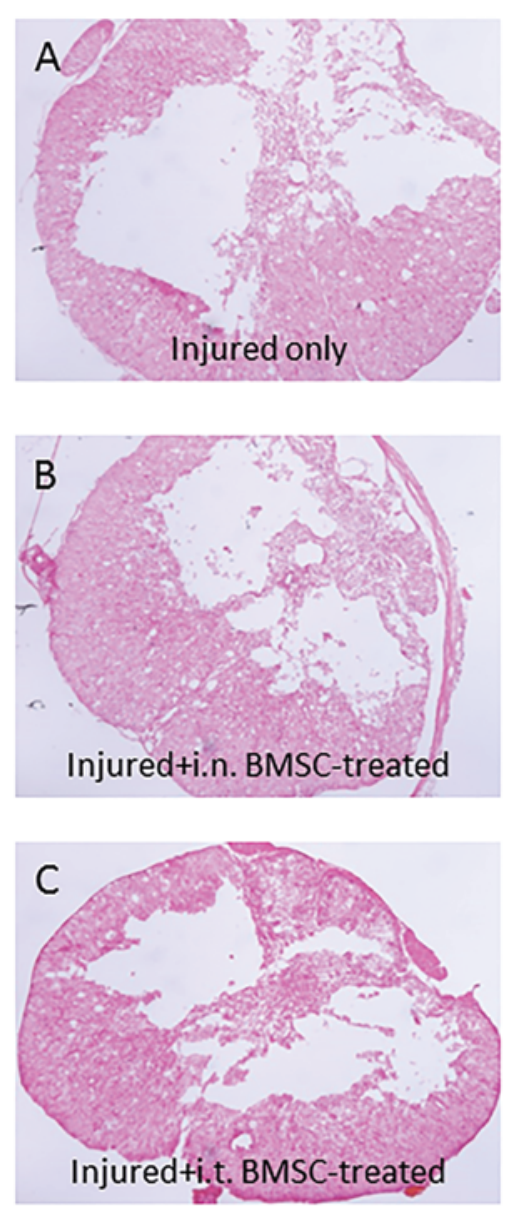
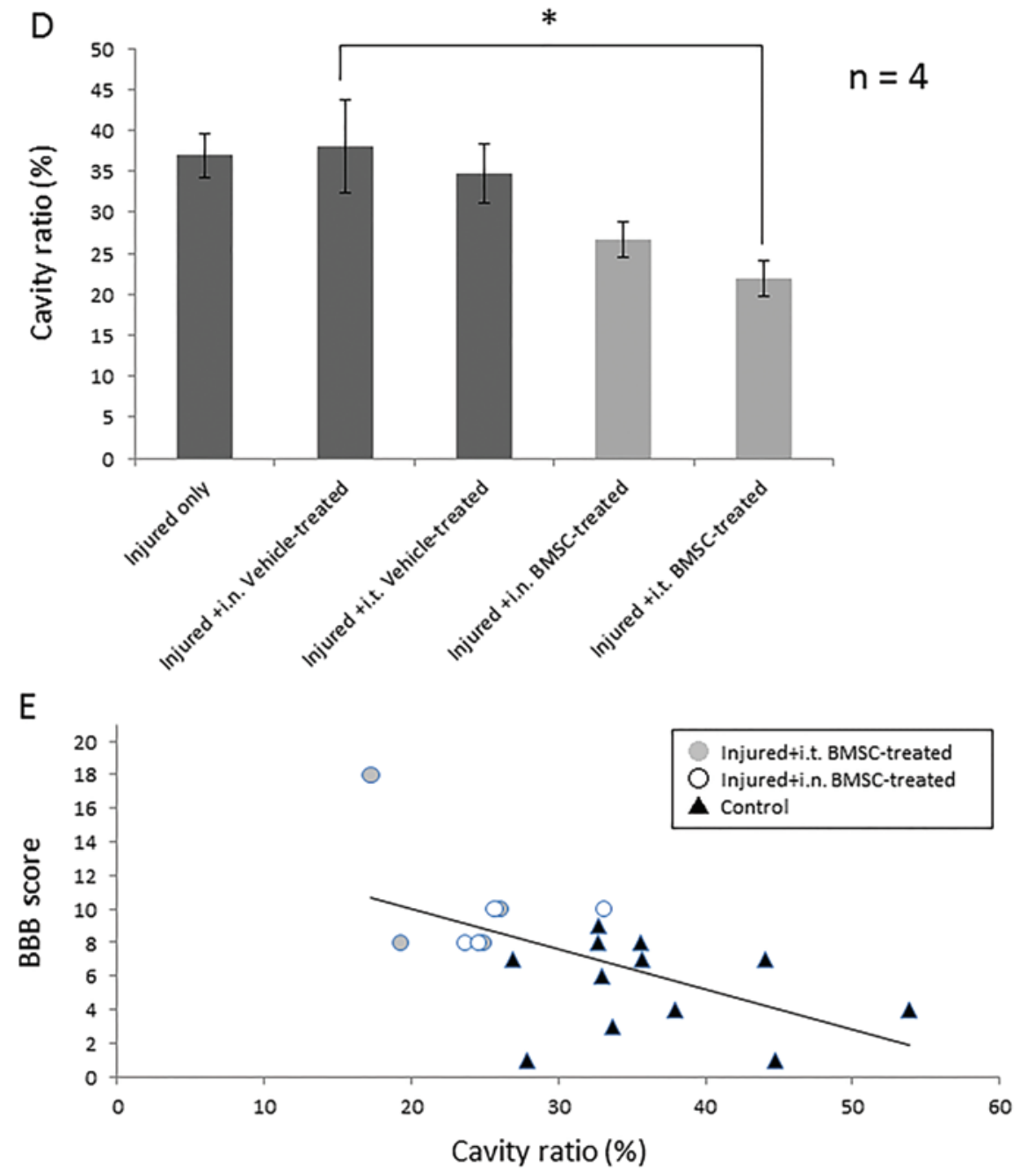

FIG. 4. A-C: Representative H \& E-stained sections of the lesion epicenter showing the cystic cavities in the 3 groups. Original magnification $\times 20$. D: Graph showing the cavity ratio of each group (mean $\pm S E M)$. A statistical difference was found only between the i.t. BMSC-treated group and the i.n. vehicle-treated group $\left({ }^{*} p<0.05\right)$. E: Graph showing the correlation between the cavity ratio and the final (4-week) BBB scores of all 20 rats. A negative significant correlation was found $\left(R^{2}=0.33, p=0.0076\right)$.

treated group, and the injured-only group were $10.55 \pm$ $6.42,9.33 \pm 2.70$, and $14.01 \pm 5.29$, respectively. However, the BDNF concentrations ( $\mathrm{pg} / \mathrm{ml})$ in the CSF of the i.n. BMSC-treated group, the i.t. BMSC-treated group, and the injured-only group were $75.25 \pm 33.65,44.98 \pm 20.12$, and $41.53 \pm 18.57$, respectively. There were no significant differences among the 3 groups with respect to NGF or BDNF levels in the CSF.

\section{Discussion}

\section{The Importance of Intranasal Cell Delivery}

The intranasal delivery of cells to the brain was first reported by Danielyan et al. ${ }^{9}$ They demonstrated it following the intranasal application of fluorescent-labeled rat MSCs or human glioma cells to naïve mice and rats. They considered 3 delivery roots: the olfactory parenchymal route after crossing the cribriform plate, the olfactory CSF route after crossing the cribriform plate, and the trigeminal CSF route through the trigeminal ganglion. ${ }^{9,10}$
Until now, the migration of intranasally administered MSCs to a lesion and the efficacy of this treatment have been reported for a cerebral infarction model in mice,,$^{12,22}$ an experimental autoimmune encephalomyelitis model in mice, ${ }^{13}$ and a Parkinson's disease model in rats. ${ }^{8}$ Surprisingly, the cells have been observed in the spinal cord as well as in the cortex, cerebellum, and brainstem 4 hours after the intranasal administration in the rat model of Parkinson's disease.

According to Danielyan et al., some factors, such as BDNF, tumor necrosis factor-alpha, and interferon-gam$\mathrm{ma}$, are thought to stimulate and regulate BMSC migration. In their review, Joyce et al..$^{14}$ noted that injected MSCs have the ability to home to the site of injury, as they are attracted to areas of hypoxia, apoptosis, or inflammation.

Thus we undertook this study to clarify whether BMSCs travel to injured spinal cord in an adult rat model. Several studies on SCI models have reported that most of the administered cells disappear from the lesion site within 1 month. ${ }^{17,19}$ Quertainmont et al. ${ }^{19}$ found no intra- 
A
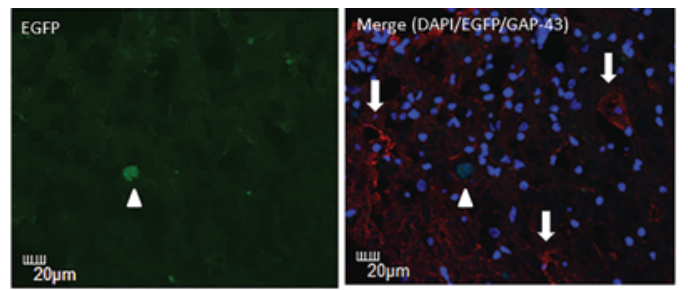

B
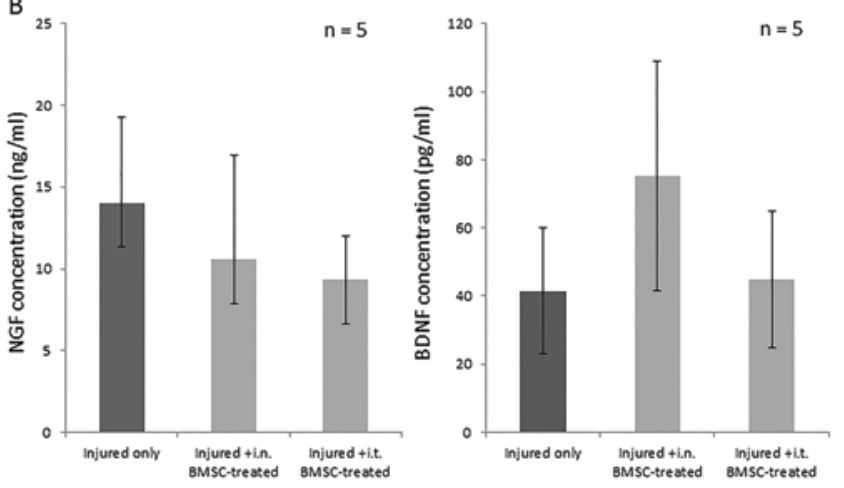

FIG. 5. A: GAP-43 (red) immunofluorescence staining of the transverse sections in the i.t. BMSC-treated group at 4 weeks. An EGFP (green)positive cell (arrowhead) was found (left), and some axonal regrowth (arrows) was confirmed around the cell (arrowhead) (right). Bar $=20$ um. B: The NGF concentration (left) and the BDNF concentration (right) in the CSF at 2 weeks are shown in the 3 groups $(n=5)$. There was no statistically significant between-groups difference with respect to neurotrophins.

venously grafted MSCs even in lesion sections examined 7 days postsurgery, although neurological improvements were seen after 1 month. Therefore, we killed half of our group of rats at 2 weeks after surgery in order to to detect intralesional cells. We killed the other half of the group 2 weeks later. Thus all the rats were killed within 1 month of surgery, which is relatively early, but we predicted that there would be some neurological recovery by this point if any were going to occur. The existence of BMSCs was confirmed in spinal cord sections obtained at both time points ( 2 and 4 weeks after surgery) in this study. We think that these findings are important because the intranasal approach is thought to be extremely favorable from the viewpoints of invasiveness and simplicity. BMSC migration was not confirmed in sections $4 \mathrm{~mm}$ from the injury epicenter, where the spinal cord appeared uninjured. Although we did not clarify whether BMSCs are transferred into the brain or CNS, our study suggests that, at least locally, BMSCs will move into an inflamed area.

\section{Cell Proportion, Limited Functional Recovery, and Possible Treatment Options With Intranasal Cell Delivery}

The proportion of intranasally administered cells delivered to the spinal cord lesion was estimated as follows. Overall, in the sections taken from rats killed 2 weeks after surgery, except for those used for cavity evaluation, there was an average of 80 BMSCs within a 570- $\mu$ m-thick cross-section. Because the diameter of the impactor tip is $2500 \mu \mathrm{m}$, approximately 350 BMSCs ([80 BSMCs $\times 2500$ $\mu \mathrm{m}] / 570 \mu \mathrm{m})$ might exist in the lesion. This accounts for $1.75 \%$ of the administered cells.
The mean BBB score of the i.n. BMSC-treated group showed significant increases as early as 1 week after surgery. However, their functional recovery was limited after 2 weeks compared with that of the i.t. BMSC-treated group. In addition, the total numbers of migrated BMSCs at both 2 weeks and 4 weeks after surgery in the i.n. BMSC-treated group were less than those of the i.t. BMSC-treated group. These findings suggest that the intrathecal application route is better than the intranasal route as far as efficiency is concerned.

In order to proceed further toward the clinical use of i.n. BMSC treatment for SCI, the timing and dosage of BMSC administration must be considered. In the present study, BMSCs penetrated to the central lesion in the i.t. BMSC-treated group in the 2-weeks section, which might influence the functional recovery. Because the i.t. BMSC-treated group had approximately 4 times as many BMSCs within the spinal cord lesion when compared with the i.n. BMSC-treated group 2 weeks postsurgery, $8 \times 10^{5}$ or more cells are recommended to be administered via the intranasal route.

With respect to the timing of the injection, Cizkova et al. ${ }^{6}$ reported the interesting finding of significantly greater motor functional recovery after 3 daily injections of a total $1.5 \times 10^{6} \mathrm{MSCs}$ via an intrathecal catheter than after a single injection of $5 \times 10^{5}$ MSCs in a rat model of contusive SCI. They attributed the greater recovery not only to an increase in the number of delivered MSCs, but also to the addition of fresh stem cells during the 3 daily applications. Furthermore, they showed different results by altering the timing of the repetitive injection.

Those finding suggest, together with our present results, that repetitive injections would be preferable, and the most effective timing should be further evaluated.

\section{The Role of BMSCs in Tissue Protection and Functional Recovery}

BMSCs are multipotent cells that have the ability to differentiate into neurons. ${ }^{16,27}$ However, the proportions of BMSCs that differentiate or survive in CNS lesions seems small. Mezey et al. have reported that no more than 5\% of grafted stromal cells differentiate into neurons within a brain lesion..$^{16}$ Others have shown the survival of less than $1 \%$ of grafted MSCs in injured spinal cord at 1 month after treatment, without any differentiation. ${ }^{3}$ Therefore, it is the neurotrophins and cytokines that are secreted from BMSCs that are believed to be much more important than the cells themselves for neuroprotection, axonal regrowth, and tissue repair; and neurotrophins and cytokines effect these outcomes via paracrine signaling..$^{14,19,20,26}$

For lesion cavity reduction and functional recovery after SCI, these mechanisms seem to act comprehensively. Neuroprotection, which is caused by reducing inflammation or preventing apoptosis, ${ }^{4}$ might occur in the acute stage after injury. Statistically significant BBB score improvements occurred in both the i.n. and i.t. BMSC-treated groups by 1 week in our study, and might suggest this action. In addition, axonal regrowth was shown within the GAP43-immunostained lesion sections in the BMSC-treated groups at 4 weeks in our study. Some studies have previously reported that the tissue repair in SCI after intrathe- 
cal administration of BMSCs was due to the proliferation of nonneural tissue, including extracellular matrices that are composed of collagen fibrils. ${ }^{17}$ In addition, others have demonstrated that the extracellular matrix that is produced by BMSCs supports neural cell growth. ${ }^{1}$

In our experiment, there was a tendency toward smaller lesion cyst size with more efficient delivery of BMSCs (i.e., greater numbers of BMSCs delivered to the lesion). Furthermore, the regression analysis of the final BBB scores and the cavity ratio clearly showed that cavity reduction had great influence on neurological functional recovery after SCI. Therefore, there was no doubt that our results also supported a tissue-sparing effect of BMSCs and their contributions to functional recovery.

\section{Evaluations of NGF and BDNF}

Neurotrophins, such as NGF and BDNF, are known to play a significant role in paracrine effects. ${ }^{7,15,25}$ NGF expression by MSCs in vitro has been shown previously, and to a lesser extent, BDNF secretion has also been confirmed. ${ }^{7,25}$

However, Quertainmont et al..$^{19}$ have reported increased NGF levels, determined by ELISA, within the injured spinal cord tissue after a MSC graft and have described the contribution of NGF to lesion repair. Furthermore, according to Donega et al., ${ }^{11}$ BDNF and NGF mRNA expression from MSCs significantly increases after co-culture with hypoxic-ischemic brain extract.

In contrast, there have been very few reports of neurotrophic factor expression in CSF after BMSC administration for CNS disease. In one report, BMSC infusion into the CSF resulted in a significant increase in neuronal density and neurite length in cultured hippocampus neurons through its trophic effects. ${ }^{17}$ According to Wang et al., ${ }^{23}$ BDNF levels in CSF were significantly higher 15 days after the intraventricular administration of BMSCs and BDNF-overexpressing BMSCs in a rat model of brain injury.

In the present study, we did not find statistically significant differences in NGF and BDNF levels in the CSF among the injured-only group and the BMSC-treated groups at 14 days. It might be worth investigating the effects of neurotrophins in an earlier period in our model because the BBB scores of the BMSC-treated groups significantly increased by 1 week postsurgery. Through further investigation, we can clarify whether NGF and BDNF in CSF can be useful biomarkers for BMSC treatment in SCI.

\section{Conclusions}

We present a new BMSC administration route for the treatment of acute SCI. The results of our study show, for the first time, BMSC migration to the spinal cord lesions in a rat model of acute SCI. However, in order to be a viable alternative to other treatment routes, this route needs further investigation. In addition, the mechanisms underlying the effectiveness of BMSCs in neurological functional recovery should be further clarified.

\section{References}

1. Aizman I, Tate CC, McGrogan M, Case CC: Extracellular matrix produced by bone marrow stromal cells and by their derivative, SB623 cells, supports neural cell growth. J Neurosci Res 87:3198-3206, 2009

2. Bakashi A, Hunter C, Swanger S, Lepore A, Fischer I: Minimally invasive delivery of stem cells for spinal cord injury: advantages of the lumbar puncture technique. J Neurosurg Spine 1:330-337, 2004

3. Boido M, Garbossa D, Fontanella M, Ducati A, Vercelli A: Mesenchymal stem cell transplantation reduces glial cyst and improves functional outcome after spinal cord compression. World Neurosurg 81:183-190, 2014

4. Caplan AI, Dennis JE: Mesenchymal stem cells as trophic mediators. J Cell Biochem 98:1076-1084, 2006

5. Chapman CD, Frey WH II, Craft S, Danielyan L, Hallschmid M, Schiöth HB, et al: Intranasal treatment of central nervous system dysfunction in humans. Pharm Res 30:2475-2484, 2013

6. Cizkova D, Novotna I, Slovinska L, Vanicky I, Jergova S, Rosocha J, et al: Repetitive intrathecal catheter delivery of bone marrow mesenchymal stromal cells improves functional recovery in a rat model of contusive spinal cord injury. $\mathbf{J}$ Neurotrauma 28:1951-1961, 2011

7. Crigler L, Robey RC, Asawachaicharn A, Gaupp D, Phinney DG: Human mesenchymal stem cell subpopulations express a variety of neuro-regulatory molecules and promote neuronal cell survival and neuritogenesis. Exp Neurol 198:54-64, 2006

8. Danielyan L, Schäfer R, von Ameln-Mayerhofer A, Bernhard F, Verleysdonk S, Buadze M, et al: Therapeutic efficacy of intranasally delivered mesenchymal stem cells in a rat model of Parkinson disease. Rejuvenation Res 14:3-16, 2011

9. Danielyan L, Schäfer R, von Ameln-Mayerhofer A, Buadze M, Geisler J, Klopfer T, et al: Intranasal delivery of cells to the brain. Eur J Cell Biol 88:315-324, 2009

10. Dhuria SV, Hanson LR, Frey WH II: Intranasal delivery to the central nervous system: mechanisms and experimental considerations. J Pharm Sci 99:1654-1673, 2010

11. Donega V, Nijboer CH, van Tilborg G, Dijkhuizen RM, Kavelaars A, Heijnen CJ: Intranasally administered mesenchymal stem cells promote a regenerative niche for repair of neonatal ischemic brain injury. Exp Neurol 261:53-64, 2014

12. Donega V, van Velthoven CT, Nijboer CH, van Bel F, Kas MJ, Kavelaars A, et al: Intranasal mesenchymal stem cell treatment for neonatal brain damage: long-term cognitive and sensorimotor improvement. PLoS ONE 8: 51253, 2013

13. Fransson M, Piras E, Wang H, Burman J, Duprez I, Harris $\mathrm{RA}$, et al: Intranasal delivery of central nervous system-retargeted human mesenchymal stromal cells prolongs treatment efficacy of experimental autoimmune encephalomyelitis. Immunology 142:431-441, 2014

14. Joyce N, Annett G, Wirthlin L, Olson S, Bauer G, Nolta JA: Mesenchymal stem cells for the treatment of neurodegenerative disease. Regen Med 5:933-946, 2010

15. Kurozumi K, Nakamura K, Tamiya T, Kawano Y, Ishii K, Kobune M, et al: Mesenchymal stem cells that produce neurotrophic factors reduce ischemic damage in the rat middle cerebral artery occlusion model. Mol Ther 11:96-104, 2005

16. Mezey E, Chandross KJ, Harta G, Maki RA, McKercher SR: Turning blood into brain: cells bearing neuronal antigens generated in vivo from bone marrow. Science 290:17791782,2000

17. Nakano N, Nakai Y, Seo TB, Homma T, Yamada Y, Ohta $\mathrm{M}$, et al: Effects of bone marrow stromal cell transplantation through CSF on the subacute and chronic spinal cord injury in rats. PLoS ONE 8:e73494, 2013

18. Osaka M, Honmou O, Murakami T, Nonaka T, Houkin K, Hamada $\mathrm{H}$, et al: Intravenous administration of mesenchymal stem cells derived from bone marrow after contusive spinal cord injury improves functional outcome. Brain Res 1343:226-235, 2010 
19. Quertainmont R, Cantinieaux D, Botman O, Sid S, Schoenen J, Franzen R: Mesenchymal stem cell graft improves recovery after spinal cord injury in adult rats through neurotrophic and pro-angiogenic actions. PLoS ONE 7:e39500, 2012

20. Sasaki M, Radtke C, Tan AM, Zhao P, Hamada H, Houkin K, et al: BDNF-hypersecreting human mesenchymal stem cells promote functional recovery, axonal sprouting, and protection of corticospinal neurons after spinal cord injury. J Neurosci 29:14932-14941, 2009

21. Syková E, Jendelová P, Urdzíková L, Lesný P, Hejcl A: Bone marrow stem cells and polymer hydrogels - two strategies for spinal cord injury repair. Cell Mol Neurobiol 26:1113-1129, 2006

22. van Velthoven CT, Sheldon RA, Kavelaars A, Derugin N, Vexler ZS, Willemen HL, et al: Mesenchymal stem cell transplantation attenuates brain injury after neonatal stroke. Stroke 44:1426-1432, 2013

23. Wang Z, Yao W, Deng Q, Zhang X, Zhang J: Protective effects of BDNF overexpression bone marrow stromal cell transplantation in rat models of traumatic brain injury. J Mol Neurosci 49:409-416, 2013

24. Wei N, Yu SP, Gu X, Taylor TM, Song D, Liu XF, et al: Delayed intranasal delivery of hypoxic-preconditioned bone marrow mesenchymal stem cells enhanced cell homing and therapeutic benefits after ischemic stroke in mice. Cell Transplant 22:977-991, 2013

25. Wilkins A, Kemp K, Ginty M, Hares K, Mallam E, Scolding $\mathrm{N}$ : Human bone marrow-derived mesenchymal stem cells secrete brain-derived neurotrophic factor which promotes neuronal survival in vitro. Stem Cell Res (Amst) 3:63-70, 2009

26. Wright KT, El Masri W, Osman A, Chowdhury J, Johnson WE: Concise review: Bone marrow for the treatment of spinal cord injury: mechanisms and clinical applications. Stem Cells 29:169-178, 2011

27. Xu H, Miki K, Ishibashi S, Inoue J, Sun L, Endo S, et al: Transplantation of neuronal cells induced from human mesenchymal stem cells improves neurological functions after stroke without cell fusion. J Neurosci Res 88:3598-3609, 2010

\section{Author Contributions}

Conception and design: Ninomiya. Acquisition of data: Ninomiya. Analysis and interpretation of data: Ninomiya. Drafting the article: Ninomiya. Reviewed submitted version of manuscript: Ohnishi. Approved the final version of the manuscript on behalf of all authors: Iwatsuki. Statistical analysis: Ninomiya. Administrative/technical/material support: Ohkawa. Study supervision: Iwatsuki, Yoshimine.

\section{Correspondence}

Koichi Iwatsuki, Department of Neurosurgery, Osaka University Graduate School of Medicine, 2-2 Yamadaoka, Suita City, Osaka, Japan, 565-0871. email: kiwatsuki@ nsurg.med.osaka-u.ac.jp. 